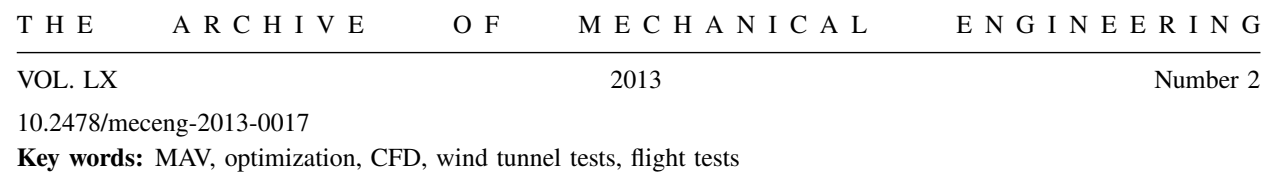

JACEK MIELOSZYK* , CEZARY GALIŃSKI*

\title{
ASSESSMENT OF THE CONCEPT OF A PROPELLER WORKING IN A SLOT IN THE MIDDLE OF WING OF A MICRO AIR VEHICLE
}

\begin{abstract}
Two configurations of micro-airplanes are compared in this paper. The first, very innovative one, has a propeller working in a slot in the middle of the wing. The second airplane has more classical configuration with a pusher propeller. Advantages and disadvantages of both configurations are evaluated, which makes it possible to assess what are the best possible mission types for each micro-airplane.
\end{abstract}

\section{Introduction}

The concept of a propeller working in a slot inside the wing has been perceived as a good concept for all weather Micro Air Vehicle (MAV). MAV is defined here as a small, light, and inexpensive unmanned flying vehicle for direct, over-the-hill reconnaissance. The focus is on fixed-wing, forward-thrust airplane, since the ability to negotiate strong opposing winds is required.

Several prototypes of fixed-wing MAVs were built to date [1-3]. They achieved good range and endurance performance. However, they suffer from near-earth boundary layer turbulence, which creates high variations in the angle of attack, as explained in [4]. A potential solution to this problem was noted in the course of the project described in [5], when one of the tested MAV configurations exhibited existence of a leading edge vortex. Leading edge vortex is a well-known phenomenon [6,7] that allows the design of super-maneuverable jet fighters, capable of flying at very high angles of attack. It was assumed that highly maneuverable MAV could be stable in the turbulent air, if equipped with a fast enough autopilot. Therefore, it was

* Institute of Aeronautics and Applied Mechanics, Warsaw University of Technology; 24 Nowowiejska Street,00-665 Warsaw, Poland; E-mail: cgalin@meil.pw.edu.pl 
decided to apply the cranked delta wing configuration, however, integration with propulsion system was not a straightforward task.

Propeller propulsion seems to be the most suitable for a fixed wing MAV. However, propeller at the vehicle front would decrease the angle of attack locally, thus annihilating the effect of the leading edge vortex. On the other hand, pusher configuration is possible to build, but it brings about the hazard of injuries for the operator during hand launching. Direct contact of the propeller with the hand of the launching person could cause personal injury, and damage the airplane. Therefore, the airplane configuration with the propeller located in the slot, inside of the wing contour (Fig. 1) was developed. In this configuration, the propeller blows directly at the control surfaces, which is perceived as an additional advantage, almost equivalent to the thrust vectoring of a modern fighter airplane.

The model of this configuration was tested in the wind tunnel as described in [8], to investigate the co-operation of the leading edge vortex with the propeller stream. First results were promising (Fig. 2) since greater lift coefficient was achieved in a wide range of angles of attack in the motor ON mode. Flow visualization confirmed this effect. Therefore, flying prototype was designed, build and flight-tested [9].

The prototype demonstrated the ability to fly controllably at extremely high angles of attack. It was possible to perform maneuvers like "cobra" and recover without altitude loss. Load factors achievable during this maneuver appeared to be greater than those calculated from wind tunnel tests (Fig. 3). Moreover, almost vertical safe landings in deep stall were also possible. These results were good enough to prove the ability of the airplane to deal with very rapid changes of the angle of attack. However, some disadvantages were also noticed. For example, the prototype was very sensitive to the motor settings. Every rpm change required immediate airplane trimming to maintain straightline flight. To solve this problem, contra rotating propeller was applied later in the project. This solution appeared successful, but it complicated the design.

Another problem was associated with manufacturability of the airplane. According to [10], wings equipped with membrane-type covering provide more stable lift coefficient and power factor in oscillating free stream. This feature was perceived as an advantage since insensitivity to the turbulence was a goal of the project. Therefore, the structure with a carbon/epoxy torsion box near the leading edge and ribs covered by membrane film at the rest of the wing was selected for the first prototype. Unfortunately, making an airplane of this structure required many man-hours, which was not acceptable for serial production. Moreover, the structure was quite delicate, which made it unsuitable for rough handling by average user. Therefore, a sandwich monocoque structure was applied in the following prototypes [11] 

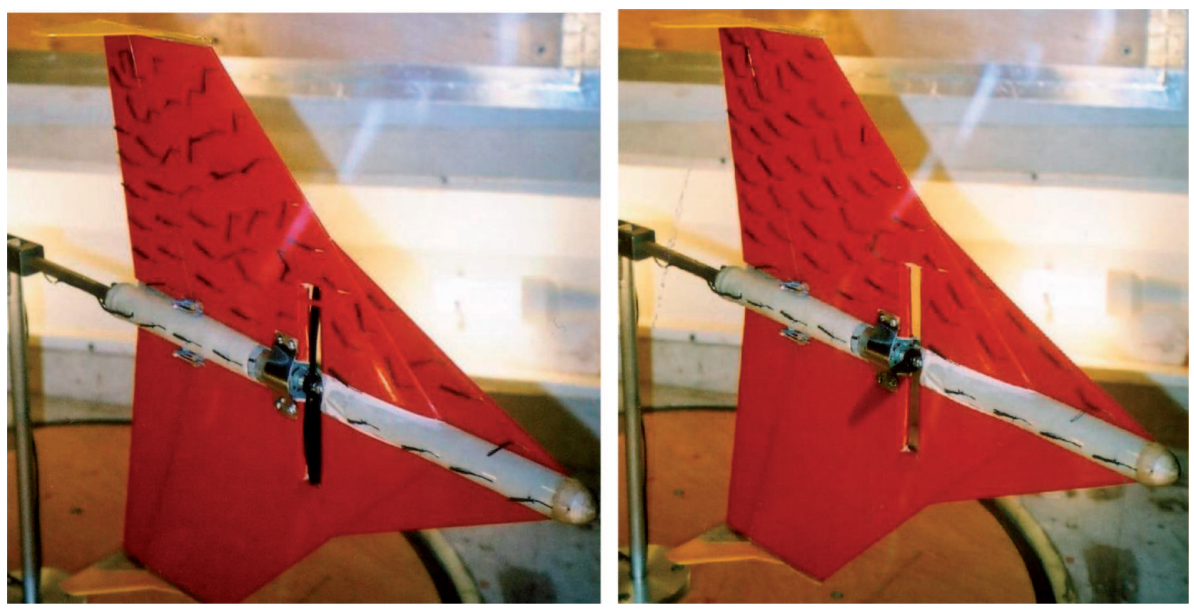

Fig. 1. Propeller effect for angle of attack of $30^{\circ}$

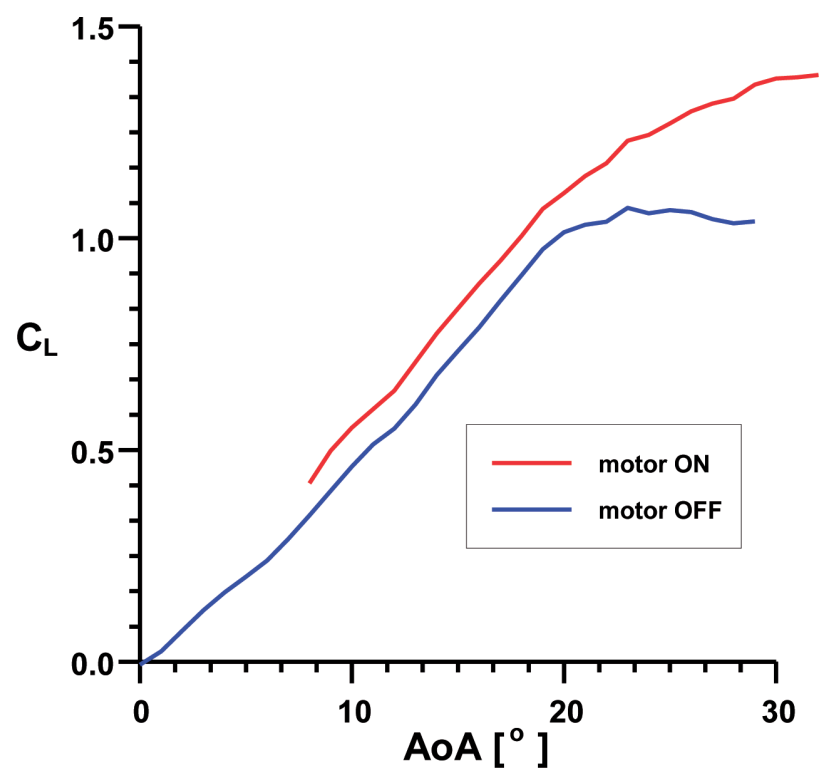

Fig. 2. Lift generated by the cranked delta wing MAV in motor ON and OFF modes, elevator in cruise position

(Fig. 4). It was less time consuming and much stronger, but also about twice heavier, which reduced cruise performance significantly. The latter feature was unsatisfactory even in the case of the first prototype, and it was even worse in the following ones. Multidisciplinary optimization was perceived as a right tool to solve this problem [12]. 


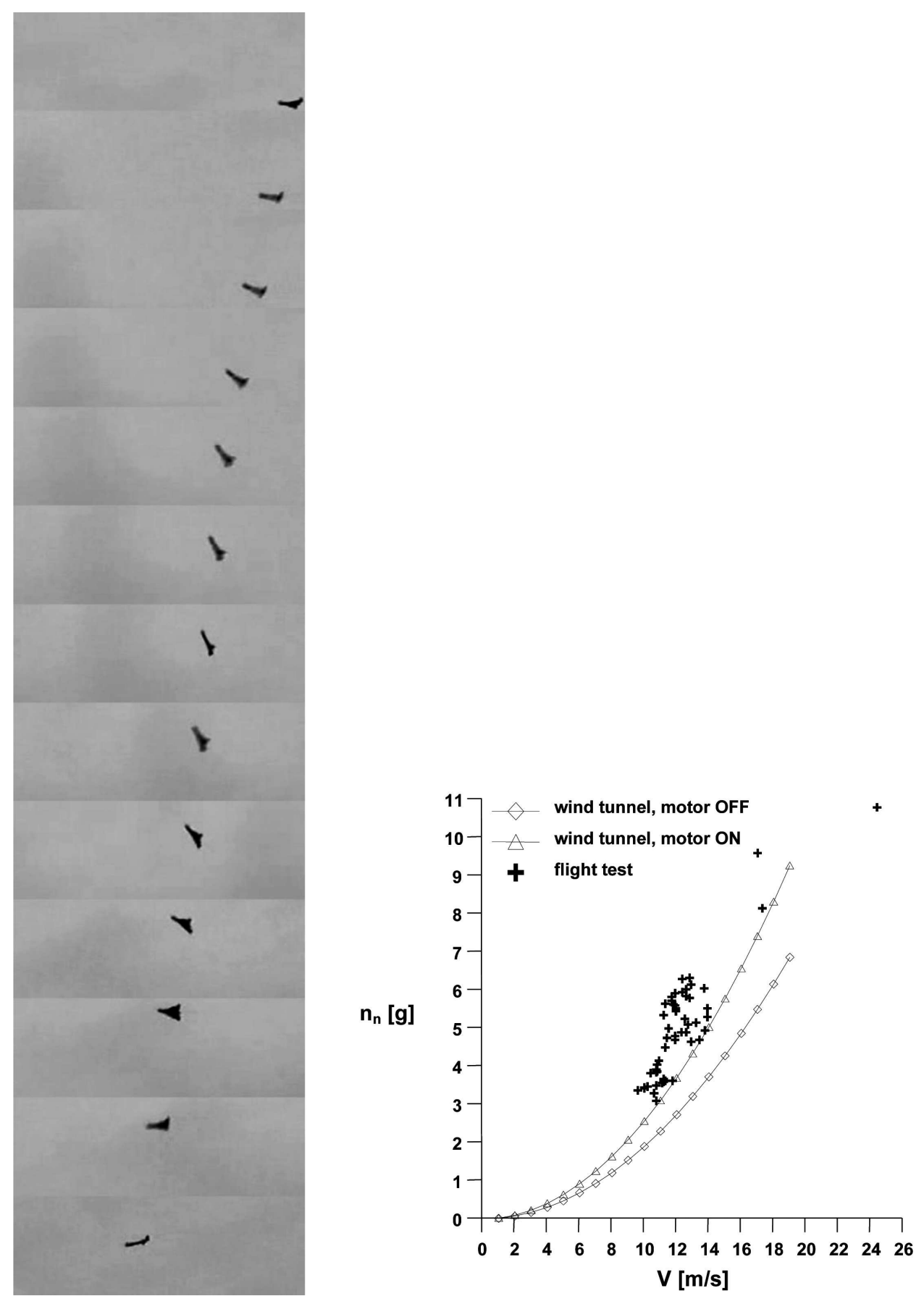

Fig. 3. Load factor acting on the MAV 

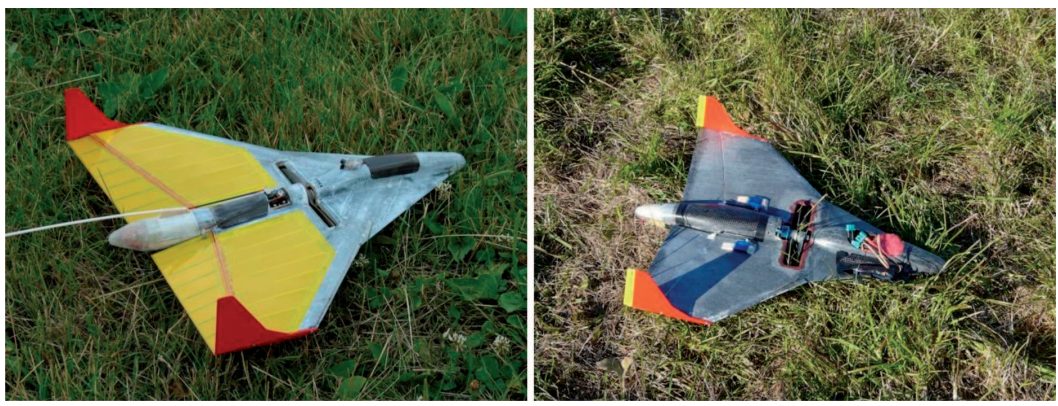

Fig. 4. First flying prototype of the MAV on the left, second prototype on the right

\section{Multidisciplinary optimization}

\subsection{D simulation}

Unsatisfactory cruise performance of the aircraft motivated the authors to look for methods of reduction of the additional drag, which was probably caused by the propeller working in the slot. 2D analyses where undertaken, with several different shapes of the slot edges, and compared with clean airfoil configuration. The performance of the stand-alone propeller was also compared. A cross section encompassing $75 \%$ of propeller's blade radius was selected for $2 \mathrm{D}$ analyses. It was assumed that the propeller is counterrotating to eliminate the roll effect strengthened by the presence of the slot. A single passages through the slot was simulated with the Fluent software, utilizing dynamic mesh features. Mesh geometry example, during one of the passages, can be seen in (Fig. 5).

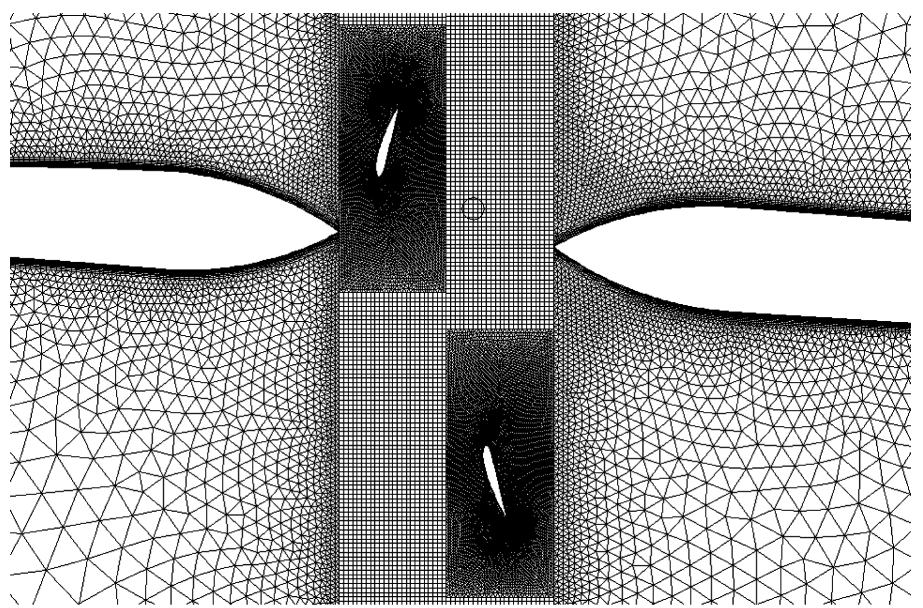

Fig. 5. 2D Grid 
Aerodynamic coefficients, integrated from the surfaces of the wing and the propeller, were averaged over the whole simulation time. Global averaged results are shown in (Fig. 6), for different types of the slot edge geometry, marked symbolically on the graph. Values on the graph are normalized to be easily comparable. The biggest change appears in aerodynamic efficiency $\mathrm{K}=\mathrm{CL} / \mathrm{CD}$, which has direct impact on UAV's flight range. The aircraft with the slot has approximately 2.5 times worse aerodynamic efficiency, but one should bear in mind that an aircraft without the slot would not have such good aerodynamic characteristics on high angles of attack (Fig. 2). This is the price for the compromise made. Worse aerodynamic efficiency results from drag increase at small angles of attack. Pitching moment, which pulls down the nose of the aircraft, is much higher for an aircraft with a slot. This disadvantageous effect also increases the trim drag of the UAV. What is interesting, the coefficients of lift and drag for propeller airfoils are both two times higher for the wing with a slot, and because of that aerodynamic efficiency of the propeller does not change. Rounded edges with quite good aerodynamic coefficients are the most promising ones, since motors in the real MAV aren't synchronized and propeller's blades can pass through the slot in any configuration.

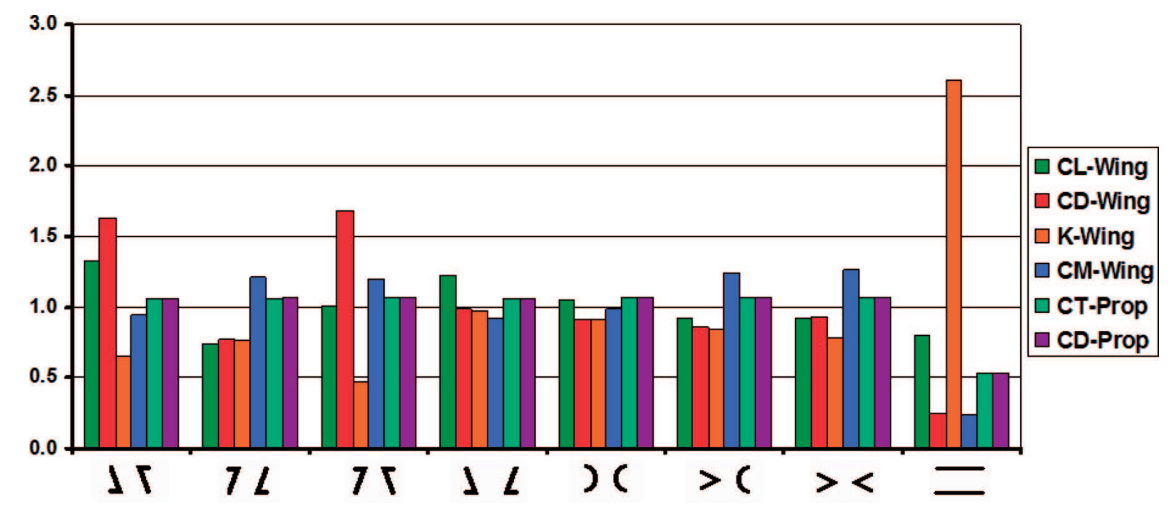

Fig. 6. Wing with slot aerodynamic coefficients

Additional investigation was undertaken for elliptical edges, which are evolution of rounded edges, to examine if it is possible to achieve a greater improvement. Several different ellipse axis ratios were tested. (Fig. 7) shows aerodynamic efficiency achieved for different proportions of the ellipses with an optimum close to the ratio equal to 0.5. Elliptical edges with a ratio of 0.5 was used for the next generation of the MAV. 


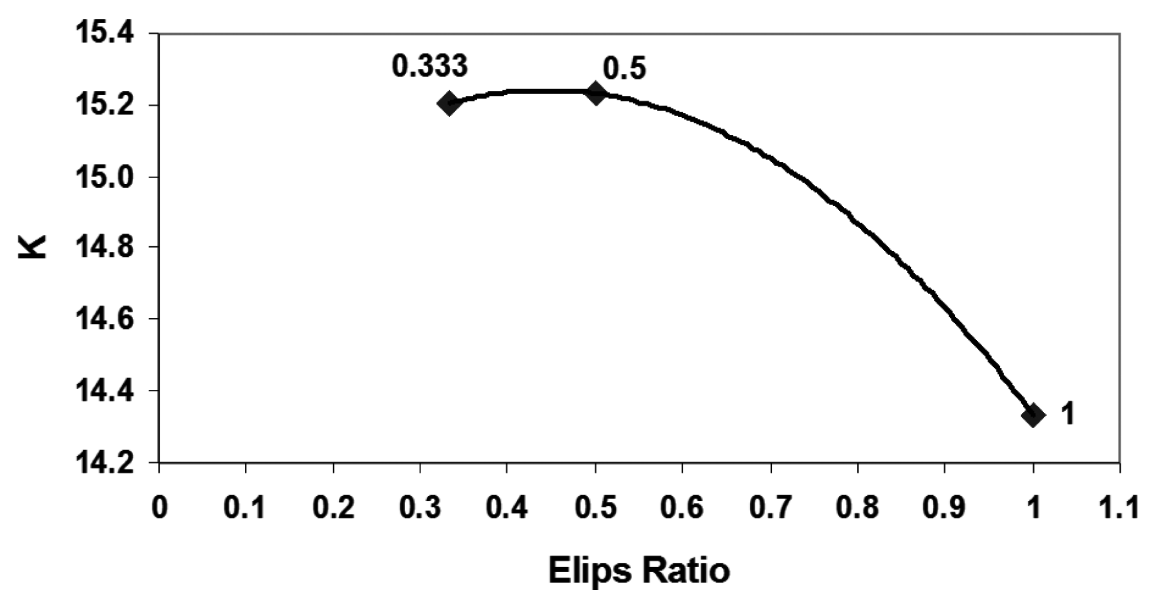

Fig. 7. Dependence of aerodynamic efficiency on elliptical edge proportions

\subsection{D optimization}

An attempt of 3D aerodynamic optimization was also undertaken after 2D simulations ware completed. A generator of highly nonlinear surface mesh of the MAV was written. A few simplifications introduced to the simulation were necessary to finish the optimization in an acceptable time. The slot in the wing was neglected, and the propeller was represented by a disc with pressure jump (Fig. 8).

The objective of the optimization was defined as minimization of the total drag for cruise conditions. Design variables were: the angle of attack, the length of the tip chord and the parameters which controlled nonlinear wing twist distribution, as well as some details of leading and trailing edge shapes. As a result of the optimization tests, the variable defining position of the center of gravity was later added.

Constrains on longitudinal static stability were set using the penalty function method to enforce obtaining real solutions. The objective function returned to the optimization algorithm was defined by equation (1), constrains on balance of vertical forces by equation (2) and constant static stability coefficient by equation (3). $\mu$ is a scalar predefined by the user.

The optimized MAV met all optimization constrains and fulfilled requirements for a bigger internal volume of equipment components. The shape of the MAV became more smooth and appeared more practical for maintenance and manufacturing with application of composite materials. However, the total drag coefficient for the cruise speed was not reduced significantly, and reached the value of 0.0595. (Fig. 8) shows the model used for optimization and the final optimized geometry, whereas (Fig. 9) presents its L/D characteristics obtained from subsequent analysis of the model with slot. It is visible 
$\mathrm{F}_{\text {objective }}=100 * \mathrm{Cx}+\mathrm{P} 1+\mathrm{P} 2$

$\mathrm{P} 1=0.5 \cdot \mathrm{C} 12 / \mu ; \mathrm{C} 1=(\mathrm{m} \cdot \mathrm{g})-(0.5 \cdot \delta \cdot \mathrm{V} 2 \cdot \mathrm{S} \cdot \mathrm{Cz})$

$\mathrm{P} 2=0.5 \cdot \mathrm{C} 22 / \mu ; \mathrm{C} 2=-0.1-\mathrm{dCm} / \mathrm{dCz}$

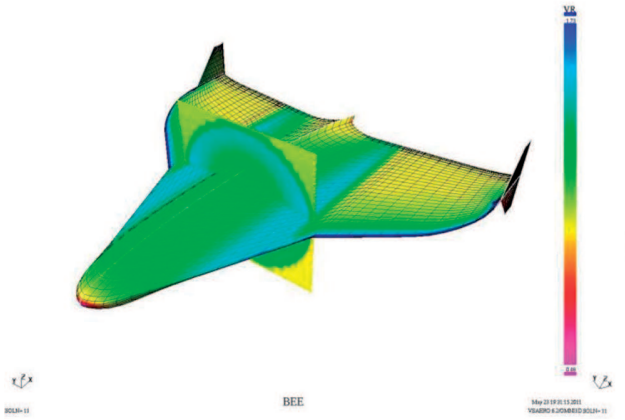

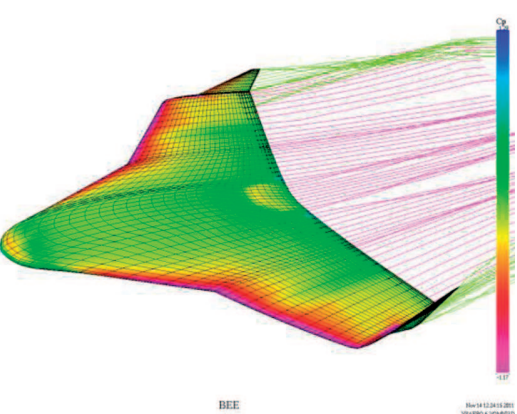

Fig. 8. On the left: initial geometry for optimization, on the right: the optimized aircraft

that maximum L/D was achieved for correct velocity, but its real value is smaller, probably due to the presence of the slot which was not considered in the model for optimization.

Demonstrators of the optimized version of the aircraft were build to prove airworthiness and measure MAV performance both in the wind tunnel and in flight [13]. The MAV was equipped with an autopilot, wireless modems with two-way communication and video system constituting a complete micro surveillance system. Integration of the system was a challenge, because the presence of additional wires made a difference.

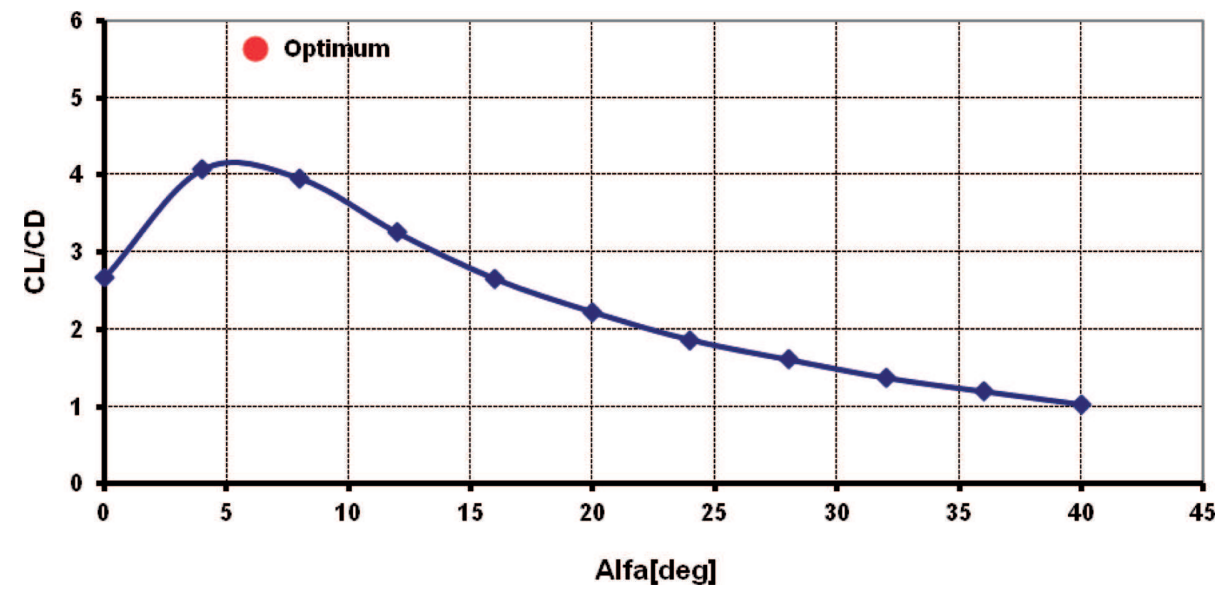

Fig. 9. Lift to drag ratio for the optimized MAV 


\section{Discussion on the propeller in the slot}

Although significant effort was devoted to the shape optimization, it was not possible to compensate for the drag penalty paid for outstanding supermaneuvrability. One must accept that an aircraft with propeller working in the slot has worse cruise performance then that in the aircraft without the slot. It is also slightly heavier since the area between the slot and the leading edge has to be strengthened to distribute stress concentration caused by the slot. Unfortunately, this complicates equipment arrangement, which is already complicated by the need of distributing equipment components in front and behind the slot. Careful equipment distribution is needed to obtain desirable $\mathrm{CG}$ position resulting from stability requirements. The presence of the slot excludes many arrangements otherwise possible. Moreover, components in front of the slot must be electrically connected with the components behind the slot. Connecting wires surround the slot and go through the very constrained space between the slot and the leading edge, shared with the reinforcing structural elements. Wires surrounding the slot are also longer then the straight ones that could be used in configuration without the slot. Finally, wires put through the constrained space complicate electromagnetic compatibility, because close proximity of wires strengthens electromagnetic interference between them. In particular, from stability point of view it is desirable to place the battery in front of the slot, since batteries are the heaviest part of equipment, which complicates the problem. Electrical motor, providing propulsion, must be located behind the slot because the space in front of the slot is occupied by autopilot which has inertial sensors in its rear part. These sensors should be as close to center of gravity of the aircraft as possible. As a result, one side of the slot is occupied by thick wires supplying power to the motor from the battery. Other wires go around in the opposite side to avoid jamming by possible high current modulation. On the other hand, placing the battery and the motor behind the slot would move the center of gravity backwards, which is not acceptable because of stability requirements. In such a case, a significant led ballast should be placed in the MAV nose, what would increase the weight of the MAV. This particular problem could be simplified if autopilot with different architecture was available. Unfortunately, it was not at the time of the design.

In consequence of all these problems, the weight of the complete vehicle became $28 \%$ greater than desirable, which made hand launch impossible, because minimum airspeed was increased due to the takeoff mass increase. The weight can be reduced by application of a lighter structure, but this also excludes hand operations since such a structure would become to delicate. This leads to the conclusion that takeoff must be performed by application 
of a mechanical launching system. This excludes the original reason for placing the propeller in the slot. On the other hand, most of the problems described above could be avoided if a conventional pusher configuration of the propulsion system was applied. This change should improve cruise performance of the MAV and reduce integration problems, but also reduce efficiency of the control surfaces, which are not overflown by high-speed slipstream from propeller any more. However, flight tests of the MAV with a slot did not reveal any problems with flying in complex weather conditions, so perhaps MAV with slightly reduced supermaneouvrability could also do the job in typical weather conditions. That is why a MAV with pusher propulsion system has also been built for comparison, and is currently flight tested.

\section{Verification of the concept}

As a prove of correctness of the concept for pusher propeller configuration, the following MAV prototype was made, still utilizing known composite technology. The first flights proved airworthiness of the pusher propeller configuration. The following advantages and disadvantages of the prototypes (Fig. 10) were observed:

Advantages and disadvantages of the MAV with pusher propeller configuration

\begin{tabular}{|l|l|}
\hline Pros: & Cons: \\
\hline$\bullet$ less integration problems & $\bullet$ reduced elevon's efficiency without inflow from propeller \\
$\bullet$ better aerodynamic performance & $\bullet$ lower maneuvering capabilities, because o lower $\mathrm{C}_{L m a x}$ \\
$\bullet$ & $\bullet$ susceptibility to roll from propeller \\
$\bullet$ & $\bullet$ some problems with aft location of CG (aft motor loca- \\
$\bullet$ lighter structure & tion) \\
$\bullet$ better Dutch roll stability & $\bullet$ possible vibrations of controls caused by closely placed \\
& propeller \\
& $\bullet$ safety during hand lunch \\
\hline
\end{tabular}

Most of the assumptions turned out to be correct. In the region where the slot was present, curvature of the plane changed rapidly and made manufacturing composite structures cumbersome, while absence of the slot solved this problem. Additionally, the slot in the wing weakened the structure in the narrowness between the leading edge and the slot (Fig. 11). Pusher configuration didn't need to be strengthened, which made the structure lighter. Its cruise performance was better, which had been already known from aerodynamic simulations.

Of course, the MAV with a pusher propeller has also disadvantages. Without inflow from propeller, the elevons have reduced efficiency. Addi- 
tionally, lower $\mathrm{C}_{\text {Lmax }}$ deteriorates maneuvering capabilities, not allowing for such rapid maneuvers as in the case of the aircraft with the slot configuration. In the case of the pusher configuration, a single propeller was used, because of the aforementioned reasons. As we expected problems with position of the center of gravity of the aircraft appeared. The motor was placed close to the center of gravity and the propeller was mounted on a long shaft going far enough behind the trialing edge of the aircraft (Fig. 12). This solution turned out to be heavy and complicated. Application of a counter-rotating propeller would lead to a solution even heavier and more complicated. This means that the propeller produces rolling moments that must be trimmed out. Contrary to what is in the counter-rotating configuration, the aircraft in the pusher configuration is less noisy.

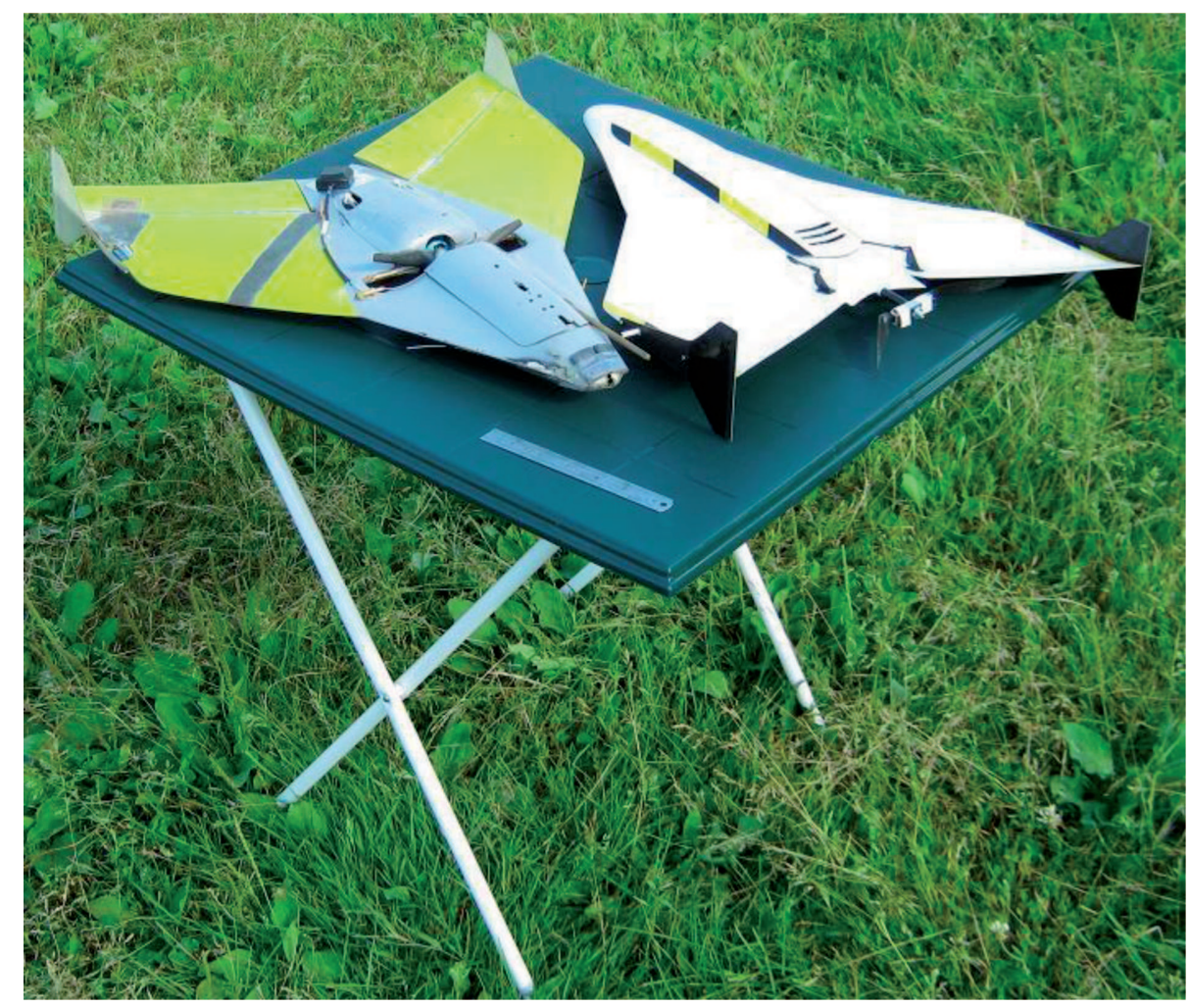

Fig. 10. The two compared prototypes 


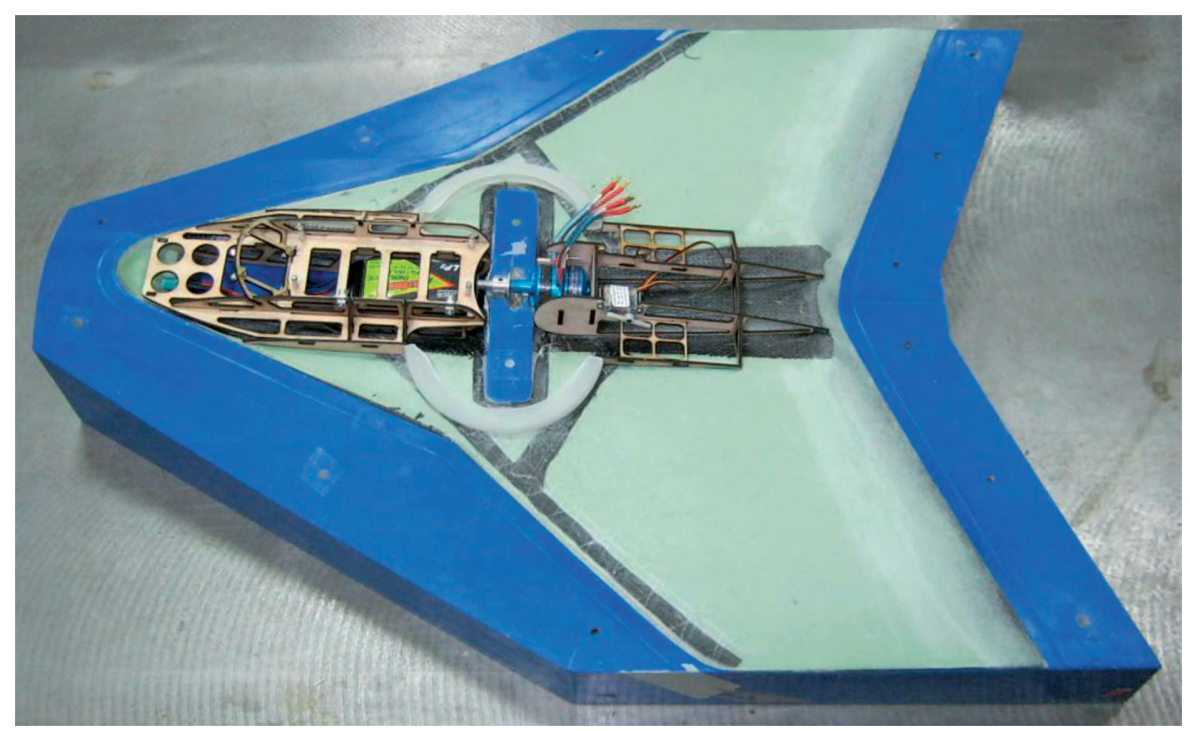

Fig. 11. Narrowness between slot and leading edge

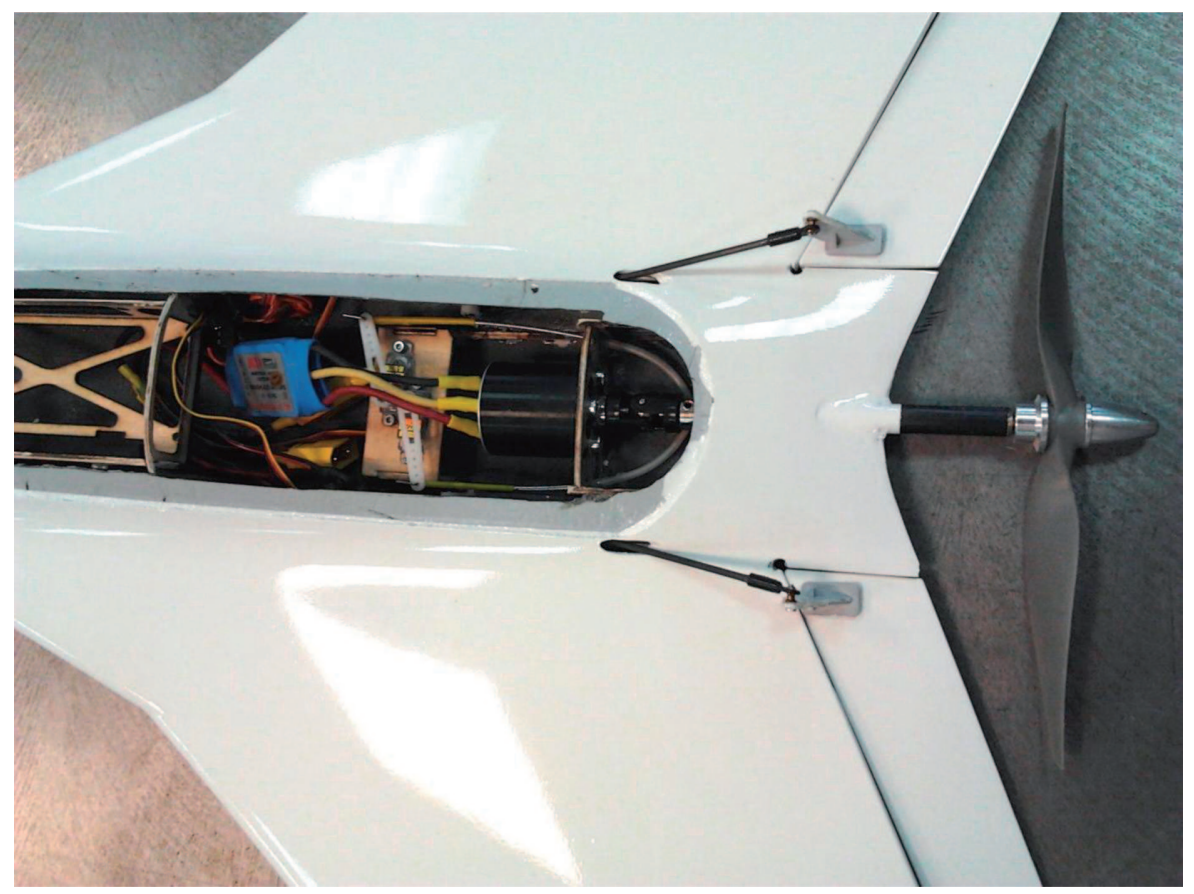

Fig. 12. Propeller on a long shaft 


\section{Conclusion}

Because of delicate structure of the MAVs and high wing loading, take off from a catapult system is desirable. Safety during possible hand lunch was the original reason for considering the propeller in the slot. However, pusher configuration aircraft is also possible, if a catapult is needed for the aircraft lunch.

Both aerodynamic configurations have their advantages and disadvantages. The slot configuration has better maneuvering capabilities and is free from rolling moments from engine thanks to the counter-rotating propeller. Unfortunately, counter-rotating propeller is more noisy. Pusher configuration has a better cruise performance and is easier to manufacture and assembly.

An aircraft with slot configuration is probably better for very specific missions where maneuvering capabilities are most important. For example, it may be important on the battlefield, where high gusts from explosions may occur. Pusher configuration is probably better for missions in more typical weather conditions, where good cruise performance is more important.

\section{Acknowledgements}

This work was supported by:

- Polish Ministry of Science and Higher Education through the grant O N509 025836

- National Science Centre (NCN) through the grant UMO-2011/01/N/ST8/07347

- Rector of Warsaw University of Technology through the grant 503R11320264004.

Special thanks for Jarosław Hajduk, who was a test pilot and to Marcin Szender, who helped to manufacture moulds.

Manuscript received by Editorial Board, January 08, 2013; final version, April 07, 2013.

\section{REFERENCES}

[1] Morris S. J.: Design and Flight Test Results for Micronized Fixed-Wing and VTOL Aircraft, Proceedings of the First International Conference on Emerging Technologies for Micro Air Vehicles, Georgia Institute of Technology, Atlanta, GA, February 1997.

[2] Bovais C., Mackrell J., Foch R., Carruthers S.: Dragon Eye UAV: Concept to Production, Proceedings of UAVs XVIII International Conference, Bristol, UK, 31 March - 2 April 2003, pp. 3.1-3.12.

[3] Grasmeyer J. M., Keennon M. T.: Development of the Black Widow Micro Air Vehicle, AIAA Paper 2001-0127, January 2001. 
[4] Watkins S., Melbourne M.: Atmospheric Winds and Their Implications for Microair Vehicles, AIAA Journal, Vol. 44, No 11, November-December 2006, pp. 2591-2600.

[5] Galiński C., Eyles M., Żbikowski R.: Experimental Aerodynamics of Delta Wing MAVs and their Scaling, Proceedings of the XVIII International UAV Conference, 31 March - 2 April 2003, Bristol, UK.

[6] Polhamus E. C.: A Concept of the Vortex Lift of Sharp-Edge Delta Wings Based on a Leading-Edge-Suction Analogy, NASA Technical Note TN D-3767, December 1966.

[7] Lamar J. E.: The use and characteristics of vortical flows near a generating aerodynamic surface: a perspective, Prog. Aerospace Sci. Vol. 34, No.3/4, 1998, pp. 167-217.

[8] Galiński C., Lawson N., Żbikowski R.: Delta wing with leading edge extension and propeller propulsion for fixed wing MAV, Proceedings of ICAS Congress, ICAS, Yokohama, Japan, 29 August - 3 September 2004, ICAS Paper 2004-1.10.5.

[9] Galiński C.: Gust resistant Fixed Wing Micro Air Vehicle, Journal of Aircraft, AIAA, Vol. 43, No. 5, September-October 2006, pp. 1586-1588.

[10] Shyy W., Klevenbring F., Milsson M., Sloan J., Carrol B., Fuentes C.: Rigid and Flexible Low Reynolds Number Airfoils, Journal of Aircraft, Vol. 36, No. 3, May-June 1999, pp. 523-529.

[11] Galiński C., Mieloszyk J., Piechna J.: Progress in the gust resistant MAV programme, 577 paper (6.10.3) in proceedings of the ICAS'2010 conference, Nice, 19-24 September 2010.

[12] Mieloszyk J.: Handling Optimization Problems on an Example of Micro UAV, pp. 1428-1438 in proceedings of the CEAS'2011 conference, Venice, 24-28 October 2011.

[13] Galiński C., Mieloszyk J.: Results of the gust resistant MAV programme, paper 186 in proceedings of the ICAS'2012 conference, Brisbane, 23-28 September 2012.

\section{Ocena koncepcji mikrosamolotu ze śmigłem w szczelinie płata}

\section{Streszczenie}

W artykule porównano dwie konfiguracje mikrosamolotów. Pierwsza z nich, innowacyjna, ma śmigło w szczelinie płata. Druga, bardziej konwencjonalna zakłada wykorzystanie śmigła pchającego. Oceniono wady i zalety obydwu konfiguracji, dzięki czemu można wskazać najbardziej odpowiednie rodzaje misji dla każdego z mikrosamolotów. 